\title{
FLIPPED EN RESPUESTA A LA DIVERSIDAD. NADIE ATRÁS, NADIE A FUERA
}

\author{
Brizeida Hernández Sánchez \\ Universidad de Salamanca \\ brizeida@usal.es \\ José Carlos Sánchez García \\ Universidad de Salamanca \\ jsanchez@usal.es \\ Greisy González Cedeño \\ Universidad Especializada de las Américas \\ greisy.gonzalez.1992@udelas.ac.pa
}

\author{
Recepción Artículo: 27 agosto 2021 \\ Admisión Evaluación: 07 septiembre 2021 \\ Informe Evaluador 1: 12 septiembre 2021 \\ Informe Evaluador 2: 13 septiembre 2021 \\ Aprobación Publicación: 15 septiembre 2021
}

\section{RESUMEN}

El mayor aprendizaje en tiempos de pandemia por la COVID-19 es que las escuelas y los estudiantes son más autodirigidos en su propia enseñanza y aprendizaje a menudo obtienen mejores resultados. La autodirección fortalece la participación de todos los estudiantes, genera autenticidad de los aprendizajes y ayuda a las escuelas a responder a mejorar las desigualdades emergentes. Con mayor respuesta en equidad y calidad para los estudiantes con riesgos vulnerables. La crisis metodológica enfrenta desacuerdos sobre qué enseñar, cómo enseñar y las implicaciones para la equidad educativa. Esta situación sin precedentes cambio los planes curriculares, el acceso a las infraestructuras de banda ancha, se legislaron políticas públicas digitales. La tecnología y el aprendizaje en línea son innovaciones enfocadas en ayudar a la cohesión, estratégica, objetivos y resultados educativos.

Este trabajo académico presenta un análisis sistemático de la investigación académica, aplicando indicadores bibliométricos y análisis conglomerados, que definen el estado actual de flipped classroom en estudiantes con necesidades educativas desde la perspectiva de la literatura. Para ello, se utilizó una base de datos de alto impacto, se analizaron un total de 567 artículos, publicados entre 2013 y 2020. La nueva modalidad de la educación a distancias nos hizo reflexionar sobre las necesidades educativas. Basado en la idea de invertir las clases, las sesiones más enriquecedoras consisten en hablar de lo leído. Es satisfactorio compartir con los estudiantes esa experiencia, con la diferencia de que el trabajo que realizamos a veces se acerca más a lo práctico, al tipo de actividades que llamamos tarea. Los hallazgos también muestran cómo este es un campo de estudio relativamente reciente de alta tendencia, con un impacto directo en todos los niveles educativos.

Palabras clave: sistemas educativos; tecnologías educativas; diversidad; flipp; equidad; participación 


\section{FLIPPED EN RESPUESTA A LA DIVERSIDAD. NADIE ATRÁS, NADIE A FUERA}

\section{ABSTRACT}

Flipped in response to diversity. No one behind, no one outside. The greatest learning in times of pandemic by COVID-19 is that schools and students are more self-directed in their own teaching and learning often get better results. Self-direction strengthens the engagement of all students, generates authenticity of learning, and helps schools respond to ameliorate emerging inequities. More responsive in equity and quality for students at vulnerable risk. The methodological crisis confronts disagreements about what to teach, how to teach and the implications for educational equity. This unprecedented situation changed curricular plans, access to broadband infrastructures, digital public policies were legislated. Technology and online learning are innovations focused on helping cohesion, strategic, educational goals and outcomes.

This academic paper presents a systematic analysis of academic research, applying bibliometric indicators and cluster analysis, which define the current state of flipped classroom in students with educational needs from the perspective of the literature. For this purpose, a high impact database was used, a total of 567 articles, published between 2013 and 2020, were analyzed. The new modality of distance education made us reflect on educational needs. Based on the idea of reversing classes, the most enriching sessions consist of talking about what we read. It is satisfying to share that experience with the students, with the difference that the work we do is sometimes closer to the practical, to the kind of activities we call homework. The findings also show how this is a relatively recent and highly trending field of study, with a direct impact on all levels of education.

Keywords: educational systems; educational technologies; diversity; flipp; equity; participation.

La pandemia causada por la COVID-19, iniciada en China y se extendió por el resto del mundo, ha obligado a un cambio violento de conductas y procedimientos institucionales en casi todos los países del mundo en lo que respecta a la economía, las manifestaciones tradicionales y políticas y también el sistema educativo académico, basado en una enseñanza presencial incompatible con una estrategia de educación a distancia; significa que la escuela se adaptan a la formación en línea, comenzaron a utilizar un enfoque de e-learning, una versión virtual para enseñar y aprender, pero también reuniones virtuales y clases invertidas basadas en el aprendizaje cooperativo e interacciones de ideas libres, reforzando una línea de investigación ya existente sobre evaluación de la eficacia de esta nueva forma de enseñanza (Ratten, 2020; Almomani et al.2021; Anafy, Jumaa, Arafa, 2021). Este estudio tiene como objetivo definir más claramente el estado del arte sobre el uso del aprendizaje invertido en la educación, es decir, ámbito académico, incluso considerando también la atención a la diversidad.

También afirmamos que los eventos recientes relacionados con la pandemia mundial, y la consiguiente limitación de movimiento y los protocolos ocasionales de bloqueo generalizados / localizados han solicitado un refuerzo de las estrategias de enseñanza combinadas. La educación es importante como intervención y prevención sanitaria, cerrar escuelas u otros centros culturales puede ser perjudicial para el desempeño cognitivo, en personas jóvenes, jóvenes o mayores (Wei, 2020; Bland et al.2021; Cordellieri et al.2021; Iqbal et al., 2021; Iodice, Cassano, Rossini, 2021). Es fundamental en este caso trabajar contra importantes prejuicios sobre el elearning y la educación invertida y si tuviéramos éxito frente a estas actitudes negativas podríamos considerar el e-learning no solo una forma necesaria de gestionar la situación actual o, en el futuro, de hacer frente a una situación particular en la que la educación a distancia es fundamental, pero el e-learning, utilizando por ejemplo el aula invertida, puede ser mejor que la educación tradicional.

Esta estrategia extensiva para todos los estudiantes ya que es un modelo pedagógico que transfiere el trabajo de determinados procesos de aprendizaje fuera del aula y utiliza el tiempo de clase, junto con la experiencia del docente, para facilitar y potenciar otros procesos de adquisición y práctica de conocimientos dentro del aula. Una visión del aprendizaje inclusivo, didáctico, metodologías colaborativas, tecnología y pedagogía inclusiva. La aplicación de metodologías activas, inductivas o hibridas en el aula. Es importante, conocer las estrategias y técnicas didácticas en el aula fuera de ella. El aprendizaje de los estudiantes, en el sentido más amplio. Los recursos tecnológicos, las redes y medios sociales y curación de contenidos. Y modelos pedagógicos: el modelo de aprendizaje inverso. A qué dedicamos el tiempo en el aula explicar contenidos, prácticas con contenidos, atender nece- 
sidades y trabajar cognitivo. Con aprender al revés los docentes en secundaria mejora la calidad de aprendizaje, el nuevo rol del profesor, cómo los estudios avalan la eficiencia de dicho método.

\section{MÉTODOS}

La revisión de la literatura y un análisis de grupos sobre el uso educativo de la clase invertida en las escuelas con estudiantes con necesidades educativas especiales. Se utilizó una cadena booleana compuesta por las palabras clave "aula invertida" relacionada con la conjunción and con la palabra clave "higher education NEE *". Usamos el "*" para incluir todas las versiones del término "alto" "education". Declaramos los siguientes criterios de inclusión: Registros que tengan en cuenta la educación relacionada con los alumnos escolares. Registros considerando educación entre estudiantes. Registros escritos en idioma inglés.

Los investigadores decidieron eligieron a jóvenes del nivel educativo secundaria obligatoria para una metodología de educación invertida u otros contextos educativos externos a las escuelas. Por el momento, solo hemos excluido 2021 como año de publicación y registros escritos en un idioma diferente al inglés, siendo el inglés el idioma predominante en las investigaciones sobre este tema (95\%). Usamos la declaración PRISMA (Liberati et al., 2009) para refinar nuestra investigación, usando SCOPUS para obtener una revisión de la literatura sobre este fenómeno. Se excluyeron muchos registros de esta revisión (285) porque no se consideraron coherentes con los objetivos de este artículo.

Fig. 1 PRISMA statement chart
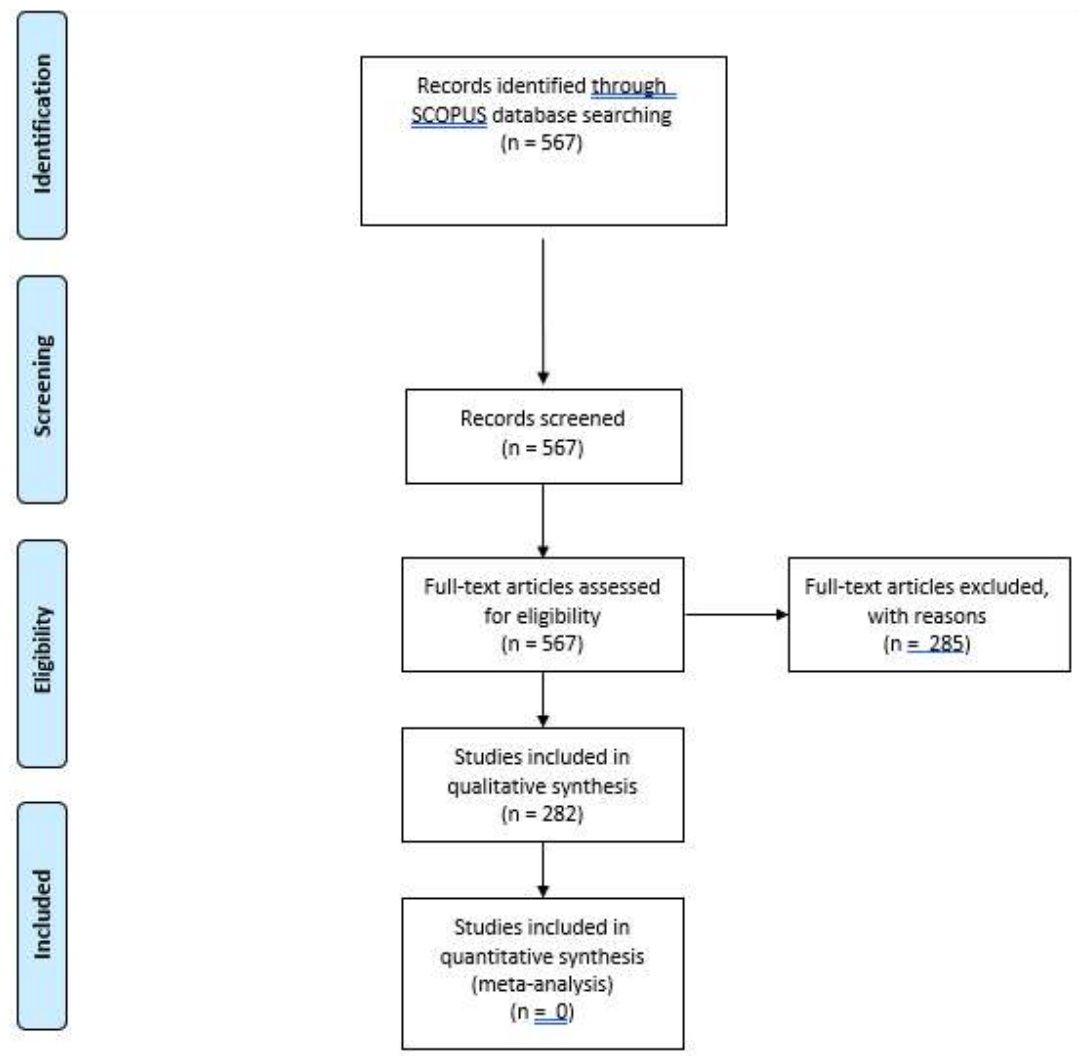


\section{FLIPPED EN RESPUESTA A LA DIVERSIDAD. NADIE ATRÁS, NADIE A FUERA}

Los artículos sobre este tema se incrementaron significativamente durante la pandemia de COVID-19 debido a la necesidad crítica de reconvertir, al menos temporalmente, el sistema educativo en una versión combinada, siendo demasiado difícil mantener una enseñanza tradicional presencial. Esperamos un crecimiento importante durante el 2020. La mayoría de los países que invierten en esta área son occidentales y países industrializados desarrollados. Los países más interesados en reforzar la educación invertida son los países en desarrollo. La mayoría de los países afectados por COVID como Estados Unidos, España, India, Rusia, países de América Latina y Turquía.

\section{RESULTADOS}

La primera mención de la técnica del aula invertida dentro de un programa educativo aparece para una aplicación de ésta en la educación médica (Meheta et al. 2013). Si consideramos años de publicación de registros sobre esta área estamos hablando de un tema de investigación reciente, donde existe un interés creciente al respecto, siguiendo una tendencia creciente constante desde 2013. Esperamos que, debido a la reciente propagación de la pandemia y la consecuente necesidad de reconvertir sistema educativo con una educación "amigable con COVID" durante la intervención de cierre, habrá un crecimiento ulterior de registros en esta área.

Fig. 2 Graphical representation of records number and publication year.

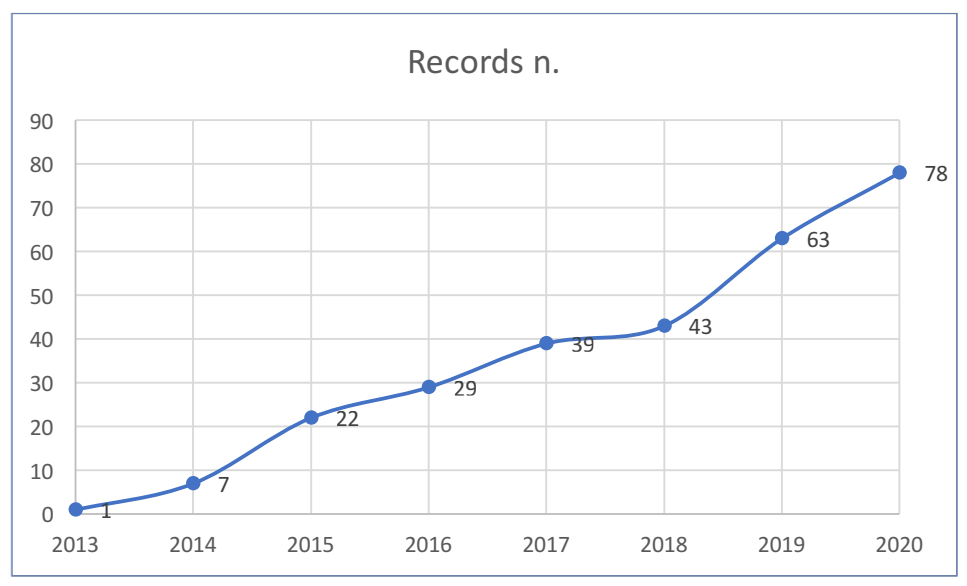

La figura 3 representa los países más productivos en este tema, donde EE.UU es el primero, de manera coherente con la mayor parte de otras áreas de investigación, pero hay que subrayar que la superioridad de la investigación de EE.UU en términos de publicaciones en este caso no es tan fuerte como en otros temas, y el Reino Unido, que suele ser el segundo país más productivo (utilizamos una investigación aleatoria rápida sobre SCOPUS durante el año pasado con diferentes palabras clave como "trastorno de aprendizaje", "escuela *", "ansiedad", "TDAH" y "TOC" y todo de estas investigaciones produjo una lista de registros donde los primeros 2 países productores de registros fueron EE. UU y el Reino Unido) es en este caso solo el quinto. Este elemento podría ser una prueba de un interés menor en la educación invertida de los investigadores del Reino Unido. 
Fig. 3 Histogram representation of 10 most prolific countries about this Research Line.

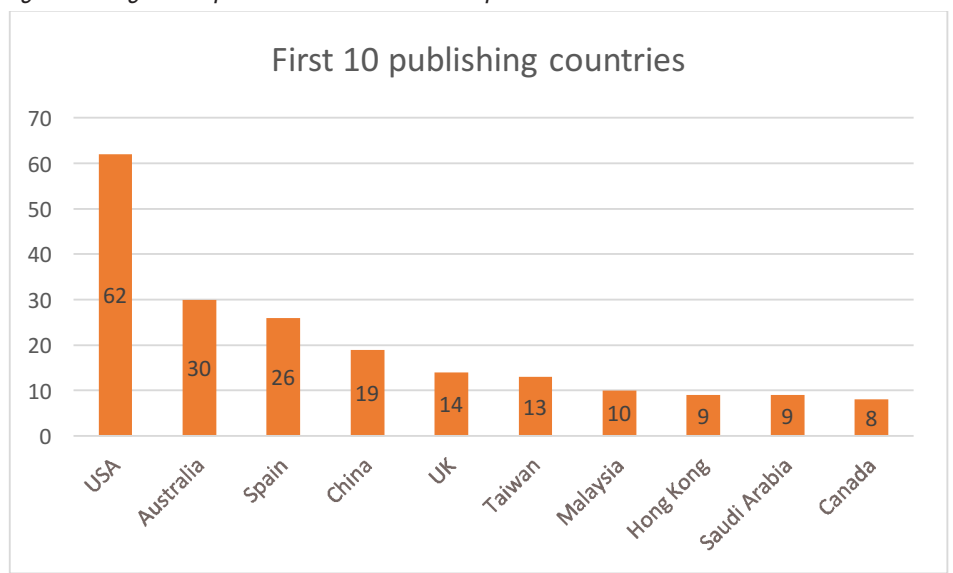

Tabla. 1 representa las revistas más activas sobre este tema. Sin considerar la última, que utiliza estrategias de aprendizaje invertido para enseñar Disciplinas Cientííicas, la mayoría de las Revistas están relacionadas con Ios Sistemas Informáticos, en los que se basa la educación invertida.

Tab 1 List of principal journals with more than 4 publication

\begin{tabular}{|c|c|c|c|}
\hline No & Journals & $\begin{array}{l}h- \\
\text { ind } \\
\text { ex }\end{array}$ & Research Area \\
\hline 11 & Computers And Education & $\begin{array}{l}16 \\
4\end{array}$ & $\begin{array}{l}\text { Computer Science, Education, E- } \\
\text { Learning }\end{array}$ \\
\hline 7 & $\begin{array}{l}\text { Computer Applications In Engineering } \\
\text { Education }\end{array}$ & 26 & $\begin{array}{l}\text { Computer Science, Engineering, } \\
\text { Social Science }\end{array}$ \\
\hline 7 & Educational Science & 7 & $\begin{array}{l}\text { Social Science, Education, } \\
\text { Developmental Psychology }\end{array}$ \\
\hline 6 & $\begin{array}{l}\text { Journal of Computing in Higher } \\
\text { Education }\end{array}$ & 31 & Social Science, Education \\
\hline 5 & Computers In Human Behavior & $\begin{array}{l}15 \\
5\end{array}$ & $\begin{array}{l}\text { Computer Science (miscellaneous), } \\
\text { Psychology (Miscellaneous), Arts } \\
\text { and Humanities (miscellaneous) }\end{array}$ \\
\hline 5 & $\begin{array}{l}\text { International Journal of Educational } \\
\text { Technology in Higher Education }\end{array}$ & 22 & $\begin{array}{l}\text { Computer Science applications, } \\
\text { Education, E-learning }\end{array}$ \\
\hline 4 & $\begin{array}{l}\text { International Journal of Management } \\
\text { Education }\end{array}$ & 22 & $\begin{array}{l}\text { Business, Management, Accounting, } \\
\text { Education }\end{array}$ \\
\hline 4 & $\begin{array}{l}\text { Journal of Advanced Oxidation } \\
\text { Technologies }\end{array}$ & 24 & Physical and Theoretical Chemistry \\
\hline
\end{tabular}

Tab. 2 muestra los 6 autores más productivos. Tienen un índice h bastante bueno son de Hong Kong, Extremadura y la Universidad de Amsterdam, respectivamente en Hong Kong, España y Holanda 3 países de los 10 más importantes sobre esta Línea de Investigación. 


\section{FLIPPED EN RESPUESTA A LA DIVERSIDAD. NADIE ATRÁS, NADIE A FUERA}

Tab 2 List of principal authors by at least 3 publications

\begin{tabular}{|c|c|c|c|c|}
\hline $\begin{array}{l} \\
o .\end{array}$ & Author & Affiliation & $\begin{array}{l}h- \\
\text { inde } \\
x\end{array}$ & Prevailing Research Area \\
\hline 5 & Hew, K., F. & $\begin{array}{l}\text { University of Hong } \\
\text { Kong }\end{array}$ & 32 & $\begin{array}{l}\text { Social Science, Computer Science, } \\
\text { Psychology, } \\
\text { Accounting }\end{array}$ \\
\hline 4 & $\begin{array}{l}\text { Gonzalez- } \\
\text { Gomez, D. }\end{array}$ & $\begin{array}{l}\text { Universidad } \\
\text { Extremadura }\end{array}$ & 27 & $\begin{array}{l}\text { Agricultural and Biological } \\
\text { Sciences, Chemistry, Social } \\
\text { Science }\end{array}$ \\
\hline 4 & Jeong, J., S. & $\begin{array}{l}\text { Universidad } \\
\text { Extremadura }\end{array}$ & 15 & $\begin{array}{l}\text { Social Science, Environmental } \\
\text { Science, Engineering }\end{array}$ \\
\hline 4 & $L o, C ., K$ & $\begin{array}{l}\text { University of Hong } \\
\text { Kong }\end{array}$ & 10 & $\begin{array}{l}\text { Social Science, Computer Scienc, } \\
\text { Business }\end{array}$ \\
\hline 3 & $\begin{array}{l}\text { Canada- } \\
\text { Canada, F. }\end{array}$ & $\begin{array}{l}\text { Universidad } \\
\text { Extremadura }\end{array}$ & 22 & $\begin{array}{l}\text { Chemistry, Social Science, } \\
\text { Environmental Science }\end{array}$ \\
\hline 3 & $\begin{array}{l}\text { Zweekhorst, } \\
\text { M.B.M. }\end{array}$ & $\begin{array}{l}\text { Amsterdam } \\
\text { University }\end{array}$ & 16 & $\begin{array}{l}\text { Medicine, Social Science, Business, } \\
\text { Nursing }\end{array}$ \\
\hline
\end{tabular}

Para tener una distribución de las principales Universidades editoriales se dividió, no solo por su continente, sino también por su área sociopolítica, por ejemplo, diferenciando Norteamérica (USA y Canadá) por CentroSudamérica (el resto de todos los países del continente americano). Decimos dividir Oriente Medio de Asia. El primero incluye a todos los países de Asia occidental, mientras que el primero no comprende el área bajo la ex URSS, se consideró a toda Rusia, incluso el área asiática, como un país europeo. Solo Oceanía y África corresponden completamente al área geográfica de sus continentes. Todo el continente africano tiene solo 2 registros sobre esta área.

Fig. 4 Distribution of publishing Universities diversified by their geopolitical and economical area.

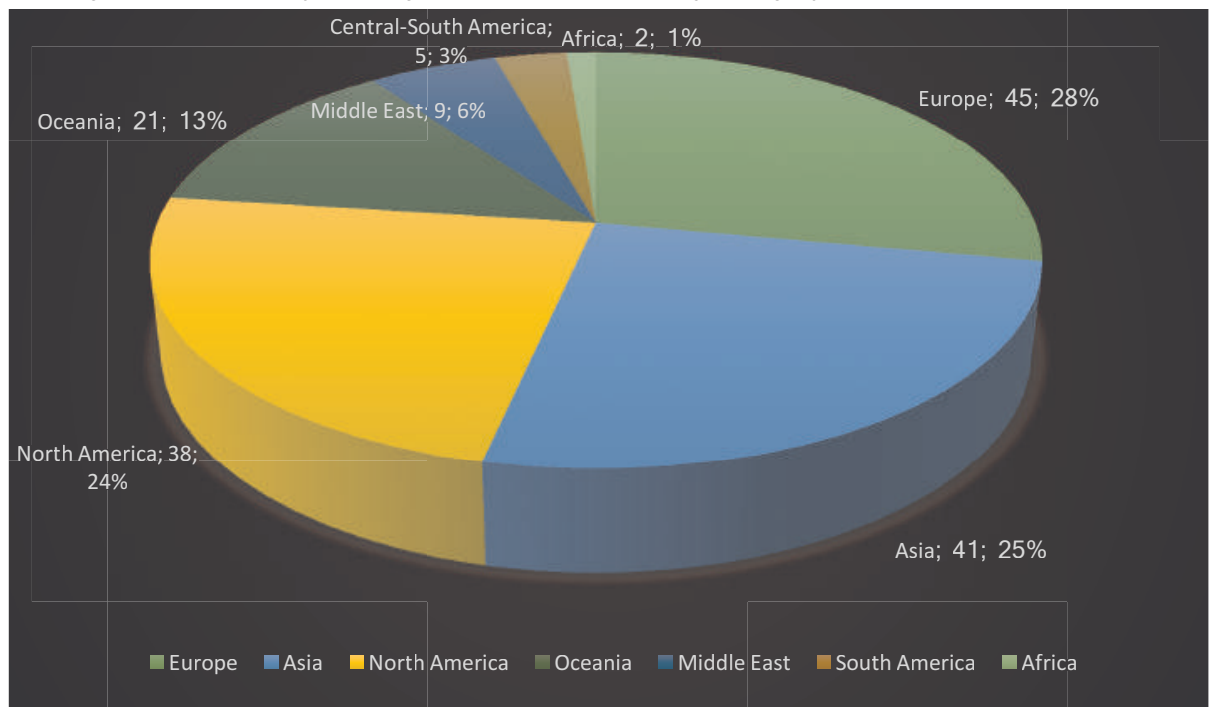




\section{Cluster analysis}

Análisis de conglomerados

Una vez refinada la lista de registros en SCOPUS usamos VosViewer (Waltman, Van Eck, Noyons, 2010) para cargar esta lista de registros creando un análisis de conglomerados, agrupando diferentes áreas de investigación sobre esta línea de investigación. El mapeo de conglomerados es una técnica de análisis importante para dar una representación gráfica de las líneas de investigación, donde los temas similares, resumidos gracias a sus palabras clave de seguimiento, se reagrupan en categorizaciones más amplias reagrupadas en cada conglomerado temático. Usando este método hemos revelado 6 clusters con 20 co-ocurrencias con 4 palabras clave como podemos apreciar en la Fig.5.

Fig. 5 Cluster mapping

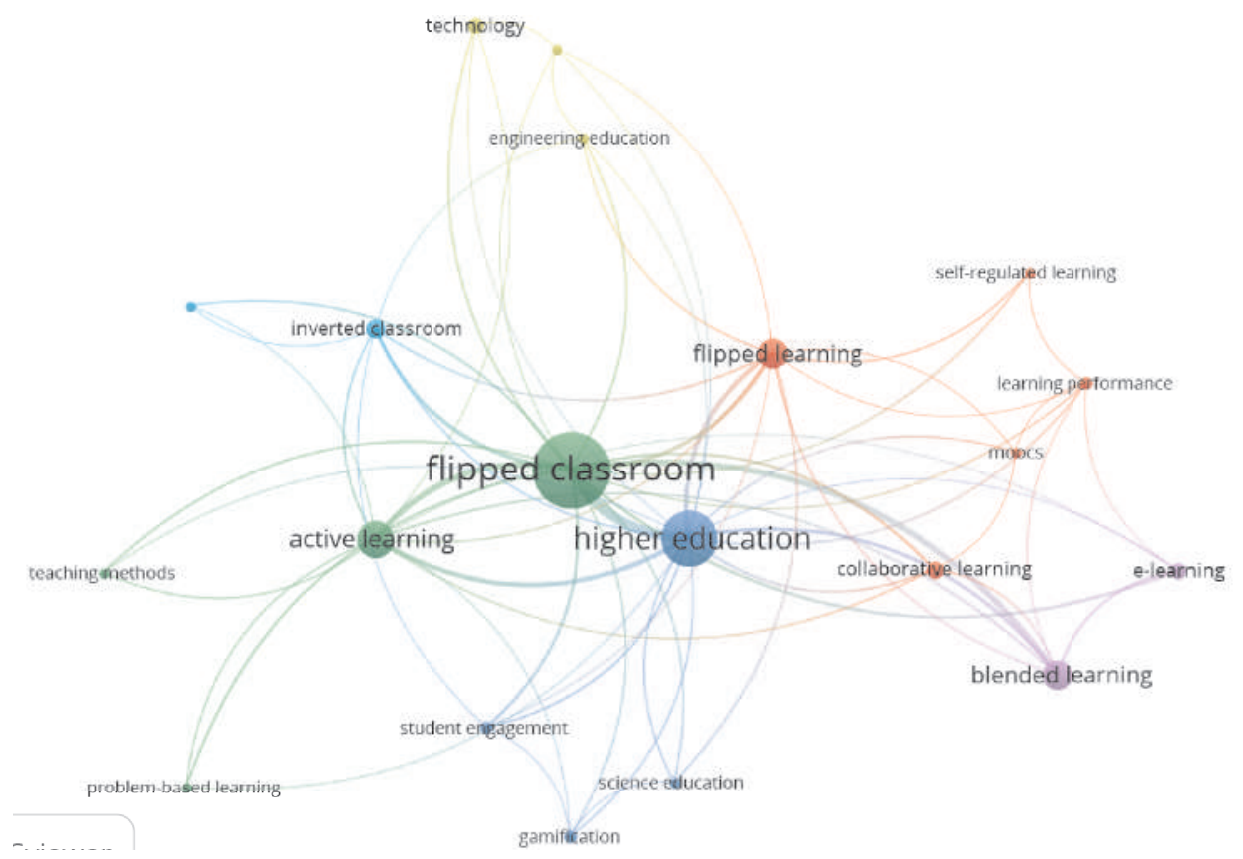

Clúster 1 (de color rojo) El primer clúster cubre el 15\% de prevalencia y se refiere a la forma en que se ha enmarcado la educación invertida en protocolos educativos internacionales precisos para formar personas, por ejemplo, con el curso en línea abierto masivo, que alcanza con eficacia a un amplio número alumnos de todo el mundo (Perez-Sanagustin et al.2017; Datsun, 2019; Wang, Zhu, 2019). El aprendizaje colaborativo (MartinezJimenez, Ruiz-Jimenez, 2020; Zheng, Johnson, Zhou, 2020) optimiza el sistema educativo y refuerza la autonomía del estudiante (Blau, Shamir-Inbal, 2017; Tikhonova, Iduganova, Lukina, 2018) y el sentido de autoaprendizaje -eficacia (Butzer, 2016; Hao, 2016; Martinez-Jimenez, Ruiz-Jimenez, 2020; Van Alten et al. 2020) en diferentes contextos de aprendizaje (Cohen et al. 2016; Evans, 2019).

Grupo 2 (de color verde) Es uno de los grupos más fuertes, con una cobertura del 66\%, a partir de dos de las palabras clave más representativas "aula invertida" (Little, 2015; Turan, Goktas, 2016; Buil-Fabrega et al. 2019) y "aprendizaje activo" (Wanner, Palmer, 2015; Novais, Silva, Muniz, 2017). La educación invertida es un método de enseñanza que puede considerarse activo, debido a la dinámica del proceso de aprendizaje, orientado 


\section{FLIPPED EN RESPUESTA A LA DIVERSIDAD. NADIE ATRÁS, NADIE A FUERA}

a la resolución de problemas específicos (Gounyou, 2015; Khan Watson, 2018; Rotrigues et al 2019) para desencadenar un nuevo tipo de relación docente entre docentes y aula con esta nueva metodología (Galway et al. 2014; Stonebaker, 2015. Este clúster está relacionado con la innovación técnica del aprendizaje invertido, dando esta novedad (Heyler, Corkill, 2015; Mennella, 2016; Salas-Rueda, 2020) boorst al sistema educativo (Mortensen, Nicholson, 2015; Sangel, 2016; Scafuto et al.2017; Niu, 2018)

Grupo 3 (de color azul) Este grupo con una cobertura del 36\% se centra en la motivación de estudio de los estudiantes, especialmente dentro de las escuelas de educación superior (O'Flaherty, Philips, 2015; Joseph, 2017; McNally et al., 2017), donde el docente tiene que captar la atención del alumno por su disciplina tanto en ciencias humanas como en otras (Khhdr, Waller, 2016; Herbert et al.2017; Cormier, Voisard, 2017; Park, Kaplan, Schlaf, 2018; Jdaitawi, 2020). Es importante mejorar la motivación de los estudiantes (Gilboy, Heinerichs, Pazzaglia, 2015; Steen-Ultheim, Foldnes, 2018; Guo, 2019; Huguet et al.2020) para alcanzar un mayor nivel de rendimiento y gamificación (Ahmad, Hinck, 2016; Forndran, Zacharias, 2019; Thongmak, 2019) es un ejemplo para reforzar la motivación, aportando aspectos lúdicos y entretenidos en el estudio.

Grupo 4 (de color amarillo) Este grupo reagrupa un subtema significativo, a pesar de su pequeña dimensión en comparación con otros grupos (9\%). Es importante porque en este caso destacamos una conexión entre tecnología y sistema educativo (Aqqal et al.2017; Sarppaje et al.2018; Park, Kaplan, Schlaf, 2018; Lo, Hew, 2019; Prevalla, Uzunboyu, 2019), donde Es importante reforzar la motivación (Thai, De Wever, Valke, 2017; Lai, Hsiao, Hsief, 2018; Rabidoux, Rottman, 2018; Portella, 2020) entre los estudiantes utilizando ambas técnicas psicológicas pero utilizando también un conocimiento técnico relacionado con la naturaleza tecnológica interna del dispositivo informático que otorga base técnica para la educación invertida (Botha-Ravyse, Blignaut, 2017; Villalba, Castilla, Redondo-Duarte, 2018; Gamez-Montero, Pena, Olmedo-Torre, 2020).

Grupo 5 (de color púrpura) Este grupo reagrupa una parte importante y bastante representativa de los registros en este estudio, alrededor del 14\% y considera el aspecto funcional principal de la educación invertida, que es ser parte de la educación combinada (Win, Wynn, 2015; Pagatpatan, Castro, 2017; Sivalapan, 2017; Thai De Wever, Valke, 2017, que alterna una interacción tradicional presencial entre el docente y el aula y un camino a distancia. La educación mixta significa ante todo un recurso Ahorro, porque el profesor y el aula no están obligados a gastar dinero y tiempo para reunirse en el mismo lugar, evitando gastar dinero en viajar, alquilar locales y ahorrar tiempo, a veces es necesario hacer frente a dificultades logísticas para moverse en algún territorio difícil. (Pagatpatan, Castro, 2017; Tikhonova, Raitskaya, 2018; García-Ponce, Mora-Pablo, 2020) 0 en situación donde es necesario dividir un aula demasiado grande (Herbert et al.2017); además de este e- el aprendizaje es una nueva forma creativa de ma aprendizaje de la edad, innovando el sistema educativo y la relación maestro-alumnos (Galway et al 2014; Al-Zaharani, 2015; Brahimi, Sarriete, 2015; Koh, 2019; Gupta, Gupta, 2020).

Grupo 6 (de color azul claro) = Ese es el grupo más pequeño (6\%), pero es importante subrayar cuánto se basa este proceso de aprendizaje en un componente tecnológico y su primera mención fue en Revistas informáticas que tratan el tema de la educación entre las disciplinas de ingeniería (Jabbar, Gasser, Lodge, 2016), utilizando la tecnología para fines educativos (Little, 2015; Engel, Heinz Sonntag, 2017; Estriègana-Valdehita, Plata, Medina-Merodio, 2017; Foster, Stagl, 2018).

\section{CONCLUSIONES}

Hay incremento en los registros sobre flipped durante los últimos años, especialmente durante 2020. La investigación sobre la educación invertida ha evolucionado y aplicado para cambiar un sistema educativo que no funciona con entornos tradicionales. Este crecimiento, referido solo a 2020, podría deberse al caso, pero es probablemente habrá un número de registros posteriores creciendo.

Se refuerza la educación invertida en países de países desarrollados encontramos dentro de los 10 países más prolíficos EE. UU., Australia y España como los 3 países con el mayor número de registros, el Reino Unido está solo en el quinto lugar y luego otro occidental. El país desarrollado es Canadá en el décimo lugar, conside- 
rando también Taiwán, Hong Kong y Malasia que son países del Este pero que pueden ser considerados como países desarrollados occidentales por su similitud económico-cultural con otros.

La educación invertida está creciendo en los países en desarrollo. Considerando la cantidad de registros, Asia es la segunda área geográfica importante que trata la educación invertida, en parte debido a la dimensión superior de este continente, en parte debido a que los primeros trabajos sobre esta área provienen de estos países, donde ya existía una fuerte tradición investigaciones sobre educación invertida en países como China y Arabia Saudita, que también forman parte de los 10 países más prolíficos. Algunos países en desarrollo importantes y poblados como Rusia y países de América del Sur faltan en este caso, no tienen una base de investigación suficientemente sólida. Estados Unidos y España tienen un gran número de registros, pero se debe a una sólida tradición investigadora previa, que acaba de reforzarse con la pandemia. En este caso podemos suponer que las dos primeras hipótesis están en su mayoría verificadas.

La relación en respuesta a la atención a la diversidad, a pesar de la crisis, ya que es una metodología didáctica que constituyen un conjunto de técnicas y formas de generar espacios de comunicación que aporten un mayor conocimiento. Invertir lo que los estudiantes suelen realizar en el aula en el caso de los estudiantes con discapacidad o necesidades educativas especiales o cualquier riesgo en el aprendizaje debe ser tomado en cuesta. Para la atención a la población con algún riesgo vulnerable y con necesidades especifica de apoyo el flipp amplía las posibilidades didácticas y, especialmente, a la distribución de recursos y actividades didácticas en la docencia sincrónica y asincrónica.

La transformación que, han asumidos los sistemas educativos, ha exigido respuesta a la población con necesidades educativas especiales desde las herramientas tecnológicas. Medidas que tratan de fomentar la participación de los estudiantes con riegos vulnerables, ha contribuido a mejorar la motivación hacia el aprendizaje. Factor importante para aprender; igual que la docencia en línea, el aula invertida puede jugar un importante papel en el proceso de enseñanza y formación a pesar de la crisis. La reacción tecnológica ha sido primordial para fomentar el acceso al conocimiento y, por tanto, una educación más inclusiva.

\section{REFERENCIAS BIBLIOGRÁFICAS}

Al-Zaharani, A., M. (2015). From passive to active: The impact of the flipped classroom through social learning platforms on higher education students' creative thinking, British Journal of Educational Technology, 46 (6), 1133-1148. Doi: 10.1111/bjet.12353.

Almomani, E., Y., Qaman, A., M., Atrooz, F., Y., Almomany, A., M., Hajjo, R., M., Almomani, H., Y. (2021). The Influence of Coronavirus Diseases 2019 (COVID-19) Pandemic and the Quarantine Practices on University Students' Beliefs About the Online Learning Experience in Jordan, Frontiers In Public Health, 8, 595874. Doi: 10.3389/fpubh.2020.595874.

Anafy, S., M., Jumaa, A., M., Arafa, M., A. (2018). A comparative study of online learning in response to the coronavirus disease 2019 pandemic versus conventional learning, Saudi Medical Journal, 41 (3), 324-331. Doi: 10.15537/smj.2021.42.3.20200741.

Aqqal, A., Elhannani, A., Haidine, A., Dahabi, A. (2017). Improving the teaching of ICT engineering using flipped learning: A personalized model and a case study, Production, 27, e20162274. Doi: 10.1590/01036513.227416

Bland, A., R., Roiser, J., P., Metha, M., A., Sahakian, B., J., Robbins, T., W., Elliot, R. (2021). The impact of COVID19 social isolation on aspects of emotional and social cognition (early access), Cognition \& Emotion. Doi: 10.1080/02699931.2021.1892593

Blau, I., Shamir-Inbal, T. (2017). Re-designed flipped learning model in an academic course: The role of co-creation and co-regulation, Computer and Education, 115, 69-81. Doi: 10.1016/j.compedu.2017.07.014

Botha-Ravyse, C., Blignaut, S. (2017). Does the early adopter catch the worm or choke on it? A reflective journey of the challenges of technology adoption in a health sciences education institution, Education for Health: Change in Learning and Practice, 30 (2), 176-181. Doi: 10.4103/efh.EfH_219_16 


\section{FLIPPED EN RESPUESTA A LA DIVERSIDAD. NADIE ATRÁS, NADIE A FUERA}

Brahimi, T., Sarirete, A. (2015). Learning outside the classroom through M00Cs, Computers in Human Behaviour, 51, 604-609. Doi: 10.1016/j.chb.2015.03.013

Buil-Fabrega, M., Casanovas, M., M., Ruiz-Munzon, N., Filho, W., L. (2019). Flipped classroom as an active learning methodology in sustainable development curricula, Suistainability, 11 (17), 4577. Doi: 10.3390/su11174577

Cohen, M., E., Poggiali, J., Lehner Quam, A., Wright, R., West, R., K. (2016). Flipping the classroom in business and education one-shot sessions: A research study, Journal of Information Literacy, 10 (2), 40-63. Doi: $10.11645 / 10.2 .2127$

Cordellieri, P., Barchielli, B., Masci, V., Viani, F., De Pinto, I., Priori, A., Torricelli, F., D., Cosmo, C., Ferracuti, S., Giannini, A., M., Burrai, J. (2021). Psychological Health Status of Psychiatric Patients Living in Treatment Communities before and during the COVID-19 Lockdown: A Brief Report, International journal of environmental research and public health, 18 (7). Doi: 10.3390/ijerph18073567

Cormier, C., Voisard, B. (2017). Flipped classroom in organic chemistry has significant effect on students' Grades, Frontiers in ICT, 4, Article number 30. Doi: 10.3389/fict.2017.00030

Datsun, N. (2019). SPOCs in university education: European experience, Voprosy Obrazovaniya, Issue 2, 182196. Doi: 10.17323/1814-9545-2019-1-162-186

Evans, L., Bosch, M., L., V., Harrington, S., Schoof, N., Coviak, C. (2019). Flipping the Classroom in Health Care Higher Education: A Systematic Review, Nurse Education, 44 (2), 74-78. Doi: 10.1097/NNE.0000000000000554

Forndran, F., Zacharias, C., R. (2019). Gamified experimental physics classes: A promising active learning methodology for higher education, European Journal of Physics, 40 (4), 045702. Doi: 10.1088/13616404/ab215e

Foster, G., Stagl, S. (2018). Design, implementation, and evaluation of an inverted (flipped) classroom model economics for sustainable education course, Journal of Cleaner Production, 183 (10), 1323-1336. Doi: 10.1016/j.jclepro.2018.02.177

Galway, L., P., Corpett, K., K., Takaro, T., K., Tairyan, K., Frank, . (2014). A novel integration of online and flipped classroom instructional models in public health higher education, BMC Medical Education, 14 (1), article number 181. Doi: 10.1186/1472-6920-14-181

Gamez, Montero, P., J., Pena, M., Olmedo-Torre, N. (2020). Flipped learning and threshold concepts in the Turbomachinery section of Fluid Engineering course (article in press). Computer Applications in Engineering Education. Doi: 10.1002/cae.22280

Garcia-Ponce, E., E., Mora-Pablo, I. (2020). Challenges of using a blended learning approach: A flipped classroom in an english teacher education program in mexico, Higher Learning Research Communications, 10 (2), 116-133. Doi: 10.18870/HLRC.V1012.1209

Guo, J. (2019). The use of an extended flipped classroom model in improving students' learning in an undergraduate course, Journal of Computing in Higher Education, 31 (2), 362-390. Doi: 10.1007/s12528-019-09224-z

Gupta, S., B., Gupta, M. (2020). Technology and e-learning in higher education, International Journal of Advanced Science and Technology, 29 (4), 1320-1325. Available at:

https://www.researchgate.net/publication/341734948_Technology_and_E-Learning_in_Higher_Education

Hao, Y. (2016). Exploring undergraduates' perspectives and flipped learning readiness in their flipped classrooms, Computers in Human Behavior, 59, 82-92. Doi: 10.1016/j.chb.2016.01.032

Herbert, C., Velan, G., M., Pryor, W., M., Kumar, R., K. (2017). A model for the use of blended learning in large group teaching sessions, BMC Medical Education, 17 (197), Article number 197. Doi: 10.1186/s12909-017$1057-2$ 
Iodice, F., Cassano, V., Rossini, P., M. (2021). Direct and indirect neurological, cognitive, and behavioral effects of COVID-19 on the healthy elderly, mild-cognitive-impairment, and Alzheimer's disease populations, Neurological Sciences, 42 (2), 455-465. Doi: 10.1007/s10072-020-04902-8

Iqbal, A., Burrin, C., Aydin, E., Beardsall, K., Wong, H., Austin, T. (2021). Generation COVID-19-Should the foetus be worried? Acta Pediatrica, 11 (3), 759-764. Doi: 10.1111/apa.15693

Jabbar, A., Gasser, R., B., Lodge, J. (2016). Can New Digital Technologies Support Parasitology Teaching and Learning? Trends in Parasitology, 32 (7), 522-530. Doi: 10.1016/j.pt.2016.04.004

Jdatawi, M (2020). Does flipped learning promote positive emotions in science education? A comparison between traditional and flipped classroom approaches, Eletronic Journal of e-Learning, 18 (18), 516-524. Doi: 10.34190/JEL.18.6.004

Joseph, D. (2017). "Surf and Turf" in higher education: An Australian music education case study, International Journal of Learning in Higher Education, 24 (1), 47-57. Doi: 10.18848/2327-7955/CGP/N24I01/47-57

Koh, J., H., L. (2019). Four pedagogical dimensions for understanding flipped classroom practices in higher education: A systematic review, Educational Sciences: Theory and Practice, 19 (4), 14-33. Doi: 10.12738/estp.2019.4.002

Khodr, M., Waller, L. (2016). Analysis of engineering students' responses to flipped classroom methodology in the United Arab Emirates, Asian Social Science, 12 (2), 93-98. Doi: 10.5539/ass.v12n2p93

Lai, H., M., Hsiao, Y., L., Hsief, P., J. (2018). The role of motivation, ability, and opportunity in university teachers' continuance use intention for flipped teaching, Computers and Education, 124, 27-50. Doi: 10.1016/j.compedu.2018.05.013

Lai, C., L., Hwang, C., J. (2016). A self-regulated flipped classroom approach to improving students' learning performance in a mathematics course, Computers and Education, 100-126-140. Doi: 10.1016/j.compedu.2016.05.006

Lee, M., S., Son, Y., E. (2014). Study of flipped learning class utilizing big data, Information, 17 (10B), 51475152. Available at:

https://www.researchgate.net/publication/297268632_Study_of_flipped_learning_class_utilizing_big_data

Liberati A., Altman D. G., Tetzlaff J., Mulrow C., Gotzsche P. C., Ioannidis J. P., et al. (2009). The PRISMA statement for reporting systematic reviews and meta-analyses of studies that evaluate healthcare interventions: explanation and elaboration. Ital. J. Public Health 7, 354-391.

Little, C. (2015). The flipped classroom in further education: literature review and case study, Research in PostCompulsory Education, 20 (3), 265-279. Doi: 10.1080/13596748.2015.1063260

Lo, C., K., Hew, K., F. (2019). The impact of flipped classrooms on student achievement in engineering education: A meta-analysis of 10 years of research, Journal of Engineering Education, 108 (4), 523-546. Doi: 10.1002/jee.20293

Meheta, N., B., Hull, A., L., Young, J., B., Stoller, J., K. (2013). Just imagine: New paradigms for medical education, Academic Medicine, 88 (10), 1418-1423. Doi: 10.1097/ACM.0b013e3182a36a07

Mennella, T. (2016). Comparing the Efficacy of Flipped vs. Alternative Active Learning in a College Genetics Course, American Biology Teacher, 78 (6), 471-479. Doi: 10.1525/abt.2016.78.6.471

Merlin-Knoblich, C., Chase, L., Smith, J., D., Opiola, K., K. (2020). A Comparison of Student Engagement in Flipped, Active Lecture, and Online Counseling Courses (article in press). Journal of Creativity in Mental Health. Doi: 10.1080/15401383.2020.1822245

Mortensen, C., J., Nicholson, A., M. (2015). The flipped classroom stimulates greater learning and is a modern 21st century approach to teaching today's undergraduates, Journal of Animal Science, 93 (7), 3722-3731. Doi: 10.2527/jas.2015-9087 


\section{FLIPPED EN RESPUESTA A LA DIVERSIDAD. NADIE ATRÁS, NADIE A FUERA}

Niu, X. (2018). Higher education pedagogical model design and application of assignments-based flipped classroom, Journal of Advanced Oxidation Technologies, 21 (2), Article number 201809089. Doi: 10.26802/jaots.2018.09089

Novais, A., S., Silva, M., B., Muniz, J., Jr. (2017). Strengths, limitations and challenges in the implementation of active learning in an undergraduate course of logistics technology, International Journal of Engineering Education, 33 (3), 1060-1069.

O'Flaherty, J., Philips, C. (2015). The use of flipped classrooms in higher education: A scoping review, Internet and higher Education, 25-85-95. Doi: 10.1016/j.iheduc.2015.02.002

Pagatpatan, J., P., Jr. Castro, J., P. (2017). Maximizing the potential of blended learning in public health education and training, Philippine Journal of Nursing, 87 (2), 26-30. Available at: https://www.researchgate.net/publication/327741894_Maximizing_the_potential_of_blended_learning_in_public_health_education_and_trai ning

Park, S., Kaplan, H., Schlaf, R. (2018). Interdisciplinary flipped learning for engineering classrooms in higher education: Students' motivational regulation and design achievement, Computer Applications in Engineering Education, 26 (3), 589-601. 10.1002/cae.21910

Park, E., 0., Park, J., H. (2018). Quasi-experimental study on the effectiveness of a flipped classroom for teaching adult health nursing, Japan Journal of Nursing Science, 15 (2), 125-134. Doi: 10.1111/jjns.12176

Perez-Sanagustin, M., Sapunar-Opazo, D., Perez-Alvarez, R., Hilliger, I., Bey, A., Maldonado-Mahauad, J., Baier, J. (2020). A M00C-based flipped experience: Scaffolding SRL strategies improves learners' time management and engagement (Article in press). Computer Applications in Engineering Education, Doi: $10.1002 /$ cae.22337

Ratten, V. (2020). Coronavirus (Covid-19) and the entrepreneurship education community, Journal of Enterprising Communities-People And Places In The Global Economy, 14 (5), 753-764. Doi: 10.1108/JEC06-2020-0121

Rodríguez, G., Diez, J., Perez, N., Banos, J., E., Carrìo, M. (2019). Flipped classroom: Fostering creative skills in undergraduate students of health sciences, Thinking Skills and Creativity, 33, 100575. Doi: 10.1016/j.tsc.2019.100575

Salas-Rueda, R., A., (2020). Flipped classroom: Pedagogical model necessary to improve the participation of the students during the learning process, LUMAT, 8 (1), 271-296. Doi: 10.31129/LUMAT.8.1.1394

Sànchez, S., P., Lopez-Belmonte, J., Moreno-Guerrero, A., J., Sola Reche, J., M., Cabrera, A., F. (2020). Effect of bring-your-own-device program on flipped learning in higher education students, Suistainability (Switzerland), 12 (9), 3729. Doi:10.3390/su12093729.

Santiago, R., \& Bergmann, J. (2018). Aprender al revés. Flipped Classroom 3.0 y Metodologías activas en el aula.

Sangel, E. (2016). To FLIP or not to FLIP: Comparative case study in higher education in Turkey, Computers in Human Behaviour, 64 (1), 547-555. Doi: 10.1016/j.chb.2016.07.034

Sarppaje, M., Jeyasala, V., M., Rathiga, K., Sasirekha, K. (2018). Flipped classroom approach to make the best utilization of esl classes at mepco schlenk engineering college - a try out, Asian ESP Journal, 14 (7), 209228.

Scafuto, I., Serra, I., Mengini, E., Maccar, E., A., Ruas, R. (2017). The impact of flipped classroom in MBA's evaluation, Education and Training, 59 (9), 914-928. Doi: 10.1108/ET-06-2016-0097

Steen-Ultheim, A., T., Foldnes, N. (2018). A qualitative investigation of student engagement in a flipped classroom, Teaching in Higher Education, 23 (3), 307-324. Doi: 10.1080/13562517.2017.1379481

Stonebaker, I. (2015). Flipping the Business Information Literacy Classroom: Redesign, Implementation, and Assessment of a Case Study, Journal of Business and Finance Librarianship, 20 (4), 283-301. Doi: 10.1080/08963568.2015.1072893 
Strayer J.F. (2012), How Learning in an Inverted Classroom Influences Cooperation, Innovation and Task Orientation, Learning Environments Research" 15, 2, pp. 171-193.

Thai, N., T., T., De Wever, B., Valke, M. (2017). The impact of a flipped classroom design on learning performance in higher education: Looking for the best "blend" of lectures and guiding questions with feedback, Computers and Education, 107 (1), 113-126. Doi: 10.1016/j.compedu.2017.01.003

Tikhonova, E., Raitskaya, L. (2018). An overview of trends and challenges in higher education on the worldwide research agenda, Journal of Language Education, 4 (4), 4-17. Doi: 10.17323/2411-7390-2018-4-4-4-7

Tikhonova, N., V., Idugonova, G., M., Lukina, M., S. (2018). Implemented teaching methods based on interactive learning process in order to increase the ability of learning foreign language, Journal of Social Sciences Research, 5, 573-578. Doi: 10.32861/jssr.spi5.473.478.

Turan, Z., Goktas, Y. (2016). The flipped classroom: Instructional efficiency and impact on achievement and cognitive load levels, Journal of E-Learning and Knowledge Society, 12 (4), 51-62.

Van Alten, D., C., D., Phielix, C., Janssen, J., Kester, L. (2020). Effects of self-regulated learning prompts in a flipped history classroom, Computers in Human Behavior, 108, 106318. Doi: 10.1016/j.chb.2020.106318.

Wei, M. (2020). Social Distancing and Lockdown - An Introvert's Paradise? An Empirical Investigation on the Association Between Introversion and the Psychological Impact of COVID19-Related Circumstantial Changes, Frontiers in Psychology, 11, 561609. Doi: 10.3389/fpsyg.2020.561609.

Win, N., L., Wynn, S., D. (2015). Introducing blended learning practices in our classrooms, Journal of Institutional Research South East Asia, 13 (2), 17-27. Available.

Zheng, X., Johnson, T.E., Zhou, C. (2020). A pilot study examining the impact of collaborative mind mapping strategy in a flipped classroom: learning achievement, self-efficacy, motivation, and students' acceptance, Educational Technology Research and Development, 68 (6), 3527-3545. Doi: 10.1007/s11423-020-09868-0. 
